\title{
Pharmacogenetic testing: 5 Questions
}

\author{
Ailyn D. Diaz, MD, and Kurt Moran, PMHNP-BC
}

Dr. Diaz is Assistant Professor of Psychiatry and Behavioral Health, Pennsylvania State University, and Faculty, Pennsylvania Psychiatric Institute, Harrisburg, Pennsylvania Mr. Moran is Instructor of Nursing, York College of Pennsylvania, York, Pennsylvania.

\section{Disclosures}

The authors report no financial relationships with any companies whose products are mentioned in this article, or with manufacturers of competing products.

doi: 10.12788/cp.0059

\section{$f$}

Discuss this article at www.facebook.com/ MDedgePsychiatry
W hen selecting a psychotropic medication for a patient with a challenging illness, you may want to consider ordering pharmacogenetic testing. By characterizing how a patient's genetic profile affects their medication metabolism, pharmacogenetic testing can potentially help improve medication adherence, reduce "trialand-error" prescribing, and target an effective treatment. Here we address 5 important questions about using pharmacogenetic testing.

\section{What can pharmacogenetic testing tell} you? Pharmacogenetic testing looks for variants in genes that can affect how a patient metabolizes specific medications. While the results will not inform you about a specific medication's effectiveness, they can describe the patient's tolerability of that medication based on his/her metabolism. Most psychotropic medications are biotransformed in the liver by cytochrome P450 (CYP) through pathway enzymes such as 2D6, 2C19, and 3A4. For example, fluoxetine and paroxetine exert their inhibition on CYP2D6, while other psychotropic medications, such as lurasidone, are metabolized at CYP3A4 and are contraindicated with potent CYP3A4 inhibitors (eg, grapefruit juice). ${ }^{1}$

In addition to CYP450 enzymes, pharmacogenetic testing can assess for the serotonin transporter gene, SLC6A4, and its sequence promoter variant, 5-HTTLPR. This sequence variation influences response to selective serotonin reuptake inhibitors, serotonin-norepinephrine reuptake inhibitors, and tertiary amine tricyclic antidepressants. ${ }^{2}$ Pharmacogenetic testing also looks for genes related to Stevens-Johnson syndrome, such as HLA-B*1502, which is associated with adverse effects of carbamazepine and lamotrigine. ${ }^{3}$ Additional testing of methylenetetrahydrofolate reductase (MTHFR) can inform you of the patient's metabolism of folic acid, which is useful for creating augmentation strategies with fluoxetine. ${ }^{4}$

\section{When should you order pharmaco-} genetic testing? Not all patients require pharmacogenetic testing. Anxious patients who have had multiple unsuccessful medication trials may be good candidates for testing. Consider testing for patients with a history of sensitivity to medications, or whose family members have experienced unusual drug responses. ${ }^{2}$

\section{What steps should you take before order- ing pharmacogenetic testing? First, obtain} your patient's informed consent, because clinical testing reveals personal genetic information. Make sure your patient understands that such testing is voluntary and that he/she can opt out. Also, explain that the information obtained from pharmacogenetic testing is confidential and will become part of the patient's medical record.

Second, choose the best test for your patient's needs. Pharmacogenetic tests can assess for single genes encoded for selected CYP450 enzymes based on pharmacokinetics (metabolism), or for multiple genes based on pharmacodynamic (mechanism of action) factors. ${ }^{5}$ In a recent randomized controlled trial, Bradley et $\mathrm{al}^{6}$ found that testing for multiple genes improved response and remission rates in patients with depression and/or anxiety.

Third, confirm that your patient's insurance covers pharmacogenetic testing, because this testing can be expensive, although some 
Pharmacogenetic testing

continued from page 42

genetic testing companies may offer patients financial assistance.

4. How are samples taken? Several methods are used for obtaining samples, including saliva, buccal swab, and peripheral blood. Your patient should not smoke, eat, or drink for at least 30 minutes before providing a saliva sample. For a buccal swab, a cotton swab is rubbed in a circular motion along the oral lining inside each of the patient's cheeks. The most invasive method is peripheral blood obtained via venipuncture. The sample is sent through expedited mail to an accredited genetic processing laboratory for analysis.

\section{How do you interpret the results?}

Pharmacogenetic testing results are provided in a confidential report. A single gene report allows you to choose psychotropic agents based on pharmacokinetics. ${ }^{5}$ Some laboratories assess multiple genes in a single report, and create categories of medications (such as "use as directed" or "use with caution") based on the pharmacodynamic factors of each agent. ${ }^{5,6}$ Certain laboratories offer dosing guidelines for types of medications that you should use with caution. ${ }^{15,6}$

When interpreting such testing results, it is critical to use your clinical judgment, because pharmacogenetic testing alone does not assess whether a medication will help improve the patient's symptoms. It is of utmost importance that you have an understanding of pharmacodynamics, knowledge of the patient's diet and age, and a caring doctor-patient relationship.

\section{References}

1. Madhusoodanan S, Velama U, Parmar J, et al. A current review of cytochrome $\mathrm{P} 450$ interactions of psychotropic drugs. Ann Clin Psychiatry. 2014;26(2):120-138.

2. Mrazek DA. Psychiatric pharmacogenomic testing in clinical practice. Dialogues Clin Neurosci. 2010;12(1):69-76.

3. Drozda K, Müller DJ, Bishop JR. Pharmacogenomic testing for neuropsychiatric drugs: current status of drug labeling, guidelines for using genetic information, and test options. Pharmacotherapy. 2014;34(2):166-184.

4. Shelton RC, Sloan Manning J, Barrentine LW, et al Assessing effects of l-methylfolate in depression management: results of a real-world patient experience trial. Prim Care Companion CNS Disord. 2013; 15(4):PCC.13m01520. doi: 10.4088/PCC.13m01520.

5. Greden JF, Parikh SV, Rothschild AJ, et al. Impact of pharmacogenomics on clinical outcomes in major depressive disorder in the GUIDED trial: a large, patientand rater-blinded randomized, controlled study. J Psychiatr Res. 2019;111:59-67.

6. Bradley P, Shiekh M, Mehra V, et al. Improved efficacy with targeted pharmacogenetic-guided treatment of patients with depression and anxiety: a randomized clinical trial demonstrating clinical utility. J Psych Res. 2018;96:100-107. 